\title{
Constructing a Predictive Model of Copper Oxidation from Experiment and Theory
}

\author{
Christopher M. Andolina ${ }^{1}$, Matthew T. Curnan ${ }^{1}$, Qing Zhu ${ }^{1}$, Wissam A. Saidi², and Judith C. Yang ${ }^{1,3}$ \\ ${ }^{1 .}$ Department of Chemical and Petroleum Engineering, University of Pittsburgh, Pittsburgh, PA (USA) \\ 2. Department of Mechanical Engineering \& Materials Science, University of Pittsburgh, Pittsburgh, PA (USA) \\ ${ }^{3}$ Department of Physics and Astronomy, University of Pittsburgh, Pittsburgh, PA (USA)
}

Corrosion costs the U.S.A. a few percent of its gross national product. Given the ubiquity of metals in modern materials and infrastructure, a comprehensive predictive model of oxidation is necessary for the tailored design of robust corrosion-resistant coatings. However, classical models of oxidation (e.g. Wagner or Cabrera-Mott theory) use the simplifying assumption of the uniform growth of metal oxides due to the lack of historical experimental tools to explore structural changes at the nanoscale. In situ environmental transmission electron microscopy (ETEM) experiments challenges these classical assumptions. Furthermore, in situ ETEM observations of metal oxidation allow for the continuous collection of data during a chemical process, in contrast to ex situ experiments, which can introduce ambiguity of results (e.g. exposure to ambient environment during specimen transfer or temporal gaps) and these ambiguous results can increase the overall costs of computational modeling. This work aims to establish a correlative investigation utilizing theory and in situ ETEM in tandem to bridge the spatial and temporal gaps between experimental and computational approaches. Initially focusing on the oxidation of copper (a well-studied model system for metal oxidation)[1], ultimately this work aspires to develop a continuous and precise predictive model of oxidation across all studied scales of measurement.

We investigated the $\mathrm{Cu}_{2} \mathrm{O}$ island nucleation preference sites (edge or terrace) using both experiment and theory for three low index $((001),(011)$, and (111)) copper film surfaces ( $70 \mathrm{~nm}$ thick) were grown via ultra-high vacuum electron-beam evaporation on a $\mathrm{NaCl}$ crystal of a specific orientation. These single crystalline $\mathrm{Cu}$ films were annealed in situ using a Hitachi H9500 ETEM equipped with a double-tilt heating holder $\left(\mathrm{T}_{\max }=1000{ }^{\circ} \mathrm{C}\right)$ - to produce faceted holes of pristine $\mathrm{Cu}$ surfaces $-\mathrm{using}$ a hydrogen atmosphere $\left(1 \times 10^{-3}\right.$ Torr $\left.\mathrm{H}_{2}\right)$. Initiation of controlled oxidation $\left(\mathrm{O}_{2}\right)$ at $350{ }^{\circ} \mathrm{C}$ was achieved using a custom built gas delivery system with three gas input lines a variety of available gases $\left(\mathrm{CH}_{4}, \mathrm{CO}_{2}, \mathrm{CO}, \mathrm{H}_{2}, \mathrm{H}_{2} \mathrm{O}\right.$, Methanol, and $\mathrm{O}_{2}$ ). Computational methods, such as climbing image nudged elastic band within density functional theory (NEB-DFT) and molecular mechanics frameworks provided a quantitative and comprehensive assessment of the relative energetics of oxygen adsorption states, activation barriers, and surface reconstructions for different facets.[2,3] The most probable diffusion pathways of oxygen and adsorption sites predict whether nucleation of $\mathrm{Cu}_{2} \mathrm{O}$ is preferred at edge or terrace sites for given surface orientations (Figure 1). Our initial results showed good agreement between the identified nucleation site preferences determined by our computational models and those observed with in situ ETEM (Figure 2). The $\mathrm{Cu}_{2} \mathrm{O}$ island density (the number of islands/ total perimeter of holes) was determined from dark field TEM images and was found to increase from $\mathrm{Cu}(001)>(011)>(111) 4.9 \times 10^{-3}, 1.5 \times 10^{-2}$, and $2.8 \times 10^{-2}$ islands/nm, respectively. Whereas island density on the terrace (number of islands/ total terrace area) increases from $\mathrm{Cu}(001)>(011)>(111) 5.0 \times 10^{-5}, 1.4 \times 10^{-4}$, and $1.7 \times 10^{-4}$ islands $/ \mathrm{nm}^{2}$, respectively. $\mathrm{Cu}_{2} \mathrm{O}$ island nucleation favors edge over terrace sites with increasing preference for $\mathrm{Cu}(001)$ compared to the $\mathrm{Cu}$ (011) and (111). Correlating these experimental data and simulations will reveal further relationships between reaction mechanisms and aid in developing a holistic understanding of how oxidation proceeds. This, in turn, will enhance the predictive power of computational models of oxidation onset [4,5], and ultimately direct future corrosion mitigation strategies, as well as the engineering of materials tuned for specific applications.[6] 
References:

[1] Q Zhu, et al. Surface Science 652 (2016), p. 98.

[2] G. Henkelman, et al. Journal of Chemical Physics 113 (2000), p.9901.

[3] Q. Zhu, et al. Journal of Physical Chemistry Letters 7 (2016), p.2530.

[4] G. Zhou, et al. Physical Review Letters 109 (2012), p.235502.

[5] Q. Zhu, et al. Journal of Physical Chemistry C 119 (2015), p.251.

[6] The authors acknowledge National Science Foundation funding, Division of Materials Research, Grants \#1508417 and 1410055. The Petersen Institute for NanoScience and Engineering Nanoscale Fabrication and Characterization Facility: Mr. Matt France and Dr. Susheng Tan.

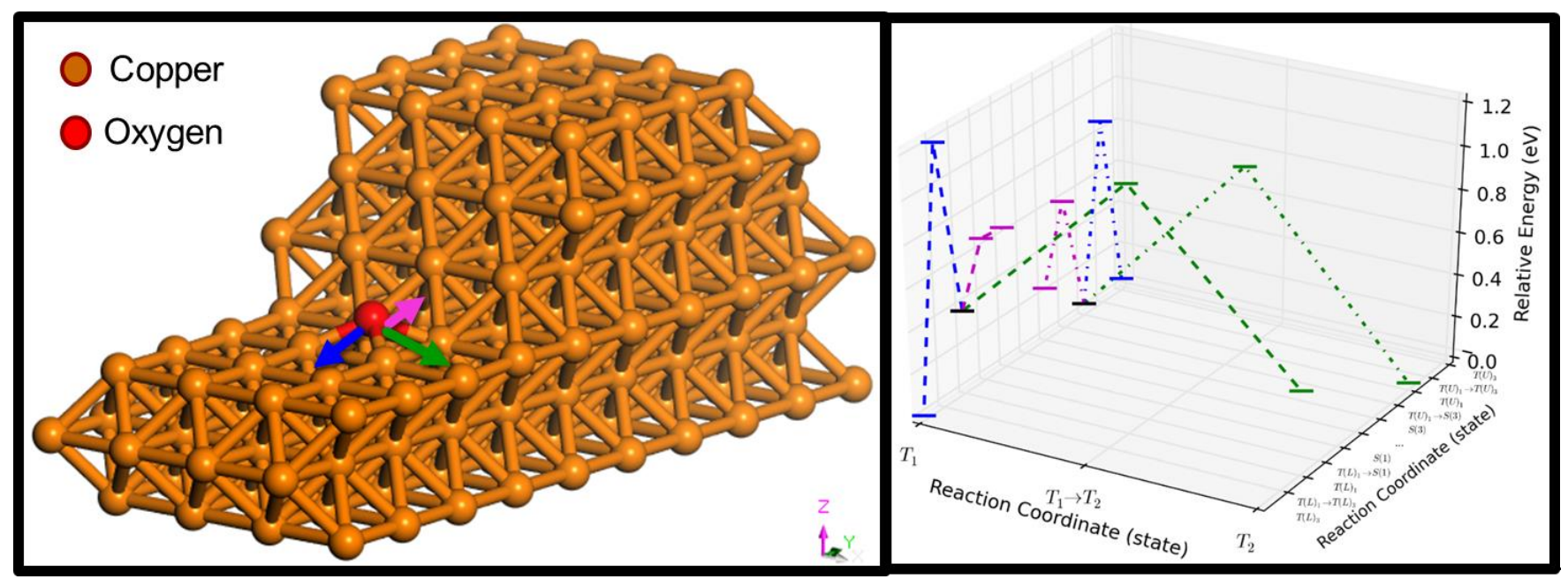

Figure 1. A visual depiction of the $\mathrm{Cu}(100)\{111\}$ facet calculation with a three step height and an $\mathrm{O}$ atom placed at the lower step edge, indicating possible step (magenta), terrace (blue), and edge (green) diffusion paths pointed to the minimum energy endpoints of nearest neighbor hops (left). Oxygen diffusion energetic barriers and states calculated using DFT-NEB in a 3D plot results indicating possible energetically favorable barriers and sites (right).

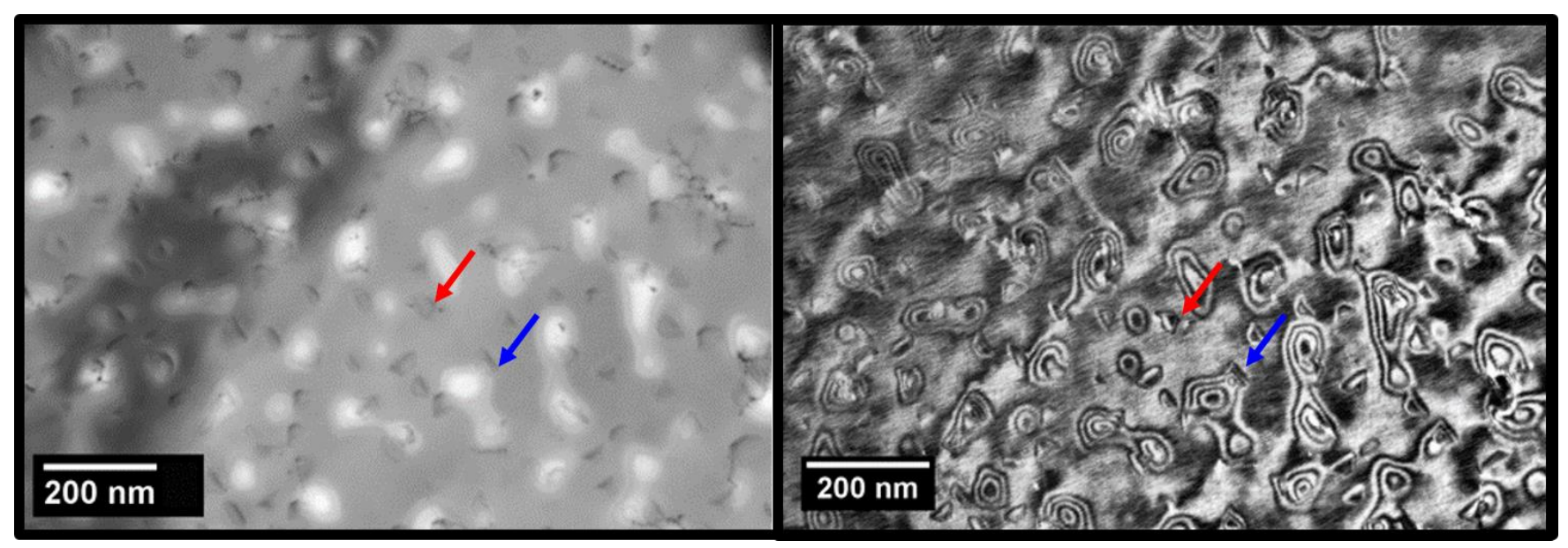

Figure 2. TEM micrographs show the location of $\mathrm{Cu}_{2} \mathrm{O}$ island nucleation on a $\mathrm{Cu}(001)$ film after in situ oxidation at $350{ }^{\circ} \mathrm{C}$ and $7.4 \times 10^{-4}$ Torr $\mathrm{O}_{2}$. Bright field TEM (left) and dark field TEM (right) showing the location of oxide islands and $\mathrm{Cu}$ film favor edge (blue) to terrace (red) sites 82 to 21 islands, respectively. 